\title{
Ventilatory management in extremely low birth weight infants
}

\author{
Shaun Morris*, Karen Choong ${ }^{\dagger}$
}

\begin{abstract}
The improvement in survival in premature infants associated with the evolution of mechanical ventilation has been accompanied by an increase in ventilator induced lung injury. High frequency ventilation has been shown to reduce the incidence of ventilator induced lung injury and hence chronic lung disease in the very low birth weight infant. The evolution in understanding how to best use high frequency ventilation in this population has prompted us to ask whether similar strategies to optimize lung volumes on conventional mechanical ventilation can minimize chronic lung disease in the neonate. We retrospectively reviewed the medical charts of 51 extremely low birth weight infants born in Kingston, Ontario in two epochs, 1990 to 1991 and 1999 to 2000, for ventilatory strategy and outcome. From our review, it is clear that surfactant therapy rapidly changes lung mechanics by improving pulmonary compliance and that lung damage may result if there are not changes in the ventilatory management to reflect the altered compliance. Early ventilation strategies during the apparently stable "honeymoon period" in a patient with respiratory distress syndrome (RDS) has significant implications on long term morbidity. In the era prior to the use of surfactant, $30 \%$ of infants died and $40 \%$ developed chronic lung disease (CLD). Immediately following the use of surfactant , mortality was reduced to $18 \%$, however, the incidence of CLD increased to $78 \%$. In the most recent era, following 10 years of experience with surfactant and mechanical ventilation, morbidity was $17 \%$ and CLD $21 \%$. This study demonstrates that a particularly crucial time is in the immediate period following surfactant administration. The use of lower peak inspiratory pressure (PIP) and mean airway pressure (MAP) over the first 24 hours and an increase in the use of synchronous intermittent mandatory ventilation (SIMV) was associated with an improved outcome. The challenge remaining is to determine how to best utilize a conventional mode of ventilation to best optimize lung volume and protect the immature lung.
\end{abstract}

\section{INTRODUCTION}

Prior to the mid 1960s, mechanical ventilators were designed to provide alveolar ventilation and additional

\footnotetext{
*To whom correspondence should be addressed: Shaun Morris, MD Resident, Department of Pediatrics, The Hospital for Sick Children, The University of Toronto shaun.morris@utoronto.ca

$\dagger$ Karen Choong, MD, Department of Pediatrics, McMaster University, McMaster Children's Hospital
}

oxygen to patients suffering from neurological impairment. In 1963, physicians at the Hospital for Sick Children in Toronto, ventilating infants with a Bird Mark VIII machine, achieved their first survivor without air leak or cerebral abnormalities (1). Soon thereafter, low levels of positive end-airway pressure (PEEP) were used to overcome the gradual decline in functional residual capacity (FRC) and minimize atelectasis. PEEP effectively stents open the lung to prevent collapse at the end expiratory point. Similarly, 
continuous positive airway pressure (CPAP) during mechanical ventilation stents open the lung throughout the entire respiratory cycle, preventing collapse and permitting lower pressures to be used for movement of gas. CPAP and PEEP became mainstays of mechanical ventilation during acute lung injury. In the early 1970s, intermittent mandatory ventilation (IMV) that delivered a certain volume at specific time intervals interspersed with the patient's respiratory efforts was the common method of neonatal ventilatory support.

Positive pressure ventilation (PPV) utilizes supraphysiologic pressues to induce the flow of gas into the lung. While PPV reduced mortality, an increase in morbidity was seen amongst survivors, in part related to ventilator management of the high pressures. In 1974, Webb and Tierney demonstrated that PPV could induce lung injury manifested by edema hemorrhage and hyaline membrane formation (2). Asynchrony between the ventilator delivered volume and spontaneous breathing is common during conventional intermittent mandatory ventilation and may result in irregular systemic and cerebral flow patterns, suboptimal gas exchange, barotraumas, airleak syndrome, pulmonary hemorrhage, intraventricular hemorrhage, periventricular leukomalacia, and chronic lung disease $(1,3)$. These complications may be avoided by sedatives or muscle relaxants to minimize the infant's spontaneous breathing. Alternatively the ventilator rate and inspiratory time may be manipulated to be synchronous with spontaneous breaths. A third approach is patient-triggered ventilation in which the breath delivered by the ventilator is triggered by the early spontaneous breathing effort. There are two types of patient-triggered ventilation: assist control, where every spontaneous breath triggers a ventilator breath and synchronized intermittent mandatory ventilation (SIMV), where the ventilator is only triggered on selected spontaneous breaths (3). Infant ventilators may use one of several methods to detect spontaneous breathing. These include abdominal movement, thoracic impedance through electrocardiogram leads, and sensors of airway pressure or flow (4). Recent advances in neonatal conventional mechanical ventilation (CMV) include increased sensitivity and hence improved patient-ventilator synchrony, pressure limited machines, graphics, calculations of gas leak, tidal volume $(\mathrm{Vt})$, minute ventilation, and flow volume loops. Additionally, impact of new methods of neonatal ventilatory management including pressure support, volume guarantee, and non-invasive ventilation such as the use of continuous positive airway pressure (CPAP) are continually being studied (5-7).

The most important advancement in neonatal care was the utilization of surfactant. Surfactant proteins were isolated and characterized in the 1970s and 1980s and the genes responsible for these proteins were cloned and sequenced (8). The clinical benefit from surfactant comes from its ability to lower alveolar surface tension and therefore increase compliance, increase stability and maintain small airway patency.

\section{"PROTECTIVE LUNG STRATEGY" - HIGH FREQUENCY OSCILLATORY VENTILATION VERSUS CONVENTIONAL VENTILATION}

High frequency oscillatory ventilation (HFOV) is a ventilatory strategy using low tidal volumes with low phasic pressure changes and supraphysiologic ventilatory frequencies. While the initial major HFOV study provided disappointing results, this has been attributed to some misunderstandings of how HFOV should be used (9). The Provo Multicenter Early HighFrequency Oscillatory Ventilation Trial concluded that when used early with a lung recruitment strategy, HFOV after surfactant replacement resulted in clinical outcomes consistent with a reduction in both acute and chronic lung injury (10). Reimensberger et al (11). managed 32 very low birth weight (VLBW) infants with first intention HFOV and compared this group to 39 historical controls managed with CMV and found that early use of HFOV with lung volume optimization significantly shortened the duration of respiratory support and improved pulmonary outcome. In a more recent study, Courtney et al. demonstrated a small but significant benefit of HFOV versus SIMV in terms of the pulmonary outcome of VLBW infants (12). In this study, HFOV did not result in an increase in the occurrence of other complications of premature birth.

It is apparent from the early use of HFOV that the outcome is dependant on the strategy applied and the individual disease process. If the lung is expanded and cyclical collapse is prevented, underlying disease process(es) may recover, limiting further inflammatory response (and hence chronic lung disease (CLD)) and potentially support endogenous surfactant production. Ventilator induced lung injury (VILI) and hence CLD may be reduced by using HFOV with a lung recruitment approach. The question then becomes: can similar results be achieved by using CMV with an "open lung" in VLBW neonates? Similarly, adult studies involving patients with acute respiratory distress syndrome and in intensive care demonstrated that protective strategies with an open lung approach (PEEP above the lower inflection point on the static pressure-volume curve, low tidal volume, permissive hypercapnia) in comparison to CMV resulted in improved survival, higher rate of weaning from mechanical ventilation and a lower rate of ventilator induced lung injury (13-15). Vazquez de Anda et al. (16) used an animal study to 
demonstrate that CMV with sufficient level of PEEP and small driving pressure amplitudes is as effective as HFOV in maintaining optimal gas exchange, improving lung mechanics, and preserving exogenous surfactant. Thome demonstrated that HFOV versus CMV with high $\mathrm{RR}$ and low peak inspiratory pressures resulted in no difference in lung injury (17). Other ventilatory factors thought to play a role in outcome are the peak inspiratory pressure (PIP) and the mean airway pressure (MAP). HFOV uses lower PIP and MAP than does CMV and recent trends have been towards lowering pressures in CMV in an effort to minimize lung trauma.

\section{PURPOSE}

There is currently minimal published Canadian data examining the ventilation experience in extremely low birth weight infants. The purpose of this study is to examine how CMV ventilation strategy has changed over time in a Canadian tertiary level neonatology unit and to determine how these changes have affected patient outcome.

\section{METHODS}

\section{Study design}

This study was conducted as a retrospective chart review of two epochs, 1990-1991 and 1999-2000. The use of surfactant in neonates with respiratory distress syndrome (RDS) was begun in Kingston, Ontario in July 1990. The eligible infants from these two epochs were assigned to one of three groups: Group 1 Immediately pre introduction of surfactant - 1990; Group 2 - Immediately post introduction of surfactant 1990-1991; Group 3 - Ten years post introduction of surfactant - 1999-2000. The two different time periods were chosen in order to examine how ventilator strategies on CMV have changed 1) following the introduction of surfactant, and 2) with time. The examination of data from both the pre and post surfactant era as well as a comparison to more recent techniques contributes to the novelty of the data presented.

\section{Patient inclusion criteria}

Eligible patients: all live-born, mechanically ventilated neonates weighing less than 1000 grams who were admitted to the neonatal intensive care unit in Kingston, Ontario during the two epochs .

\section{Data collection}

For all eligible infants, the ventilator settings were recorded for the first 72 hours of life. The median value of the ventilator settings was calculated over the first three 24 hour periods of life and the ranges and standard deviations were recorded. Outcomes of interest were:
1) Ventilator settings during hours 0 - 72 of life

2) Incidence of bronchopulmonary dysplasia (BPD) as defined by use of supplemental oxygen at $36 / 40$ weeks corrected gestational age

3 ) Total duration of $\mathrm{O} 2$ dependence

4) Total duration of mechanical ventilation

5) Incidence of severe intraventricular hemorrhage (i.e. grade III/IV)

6) NICU mortality.

\section{STATISTICAL ANALYSIS}

An unpaired t-test was used for parametric data. Categorical data were analysed by means of a Fisher's exact test or Chi2 analysis as appropriate. For all testing, a $p$ value of $<0.05$ was accepted as significant. Comparisons were made between:

1) Group 1 and Group 2

2) Group 2 and Group 3

\section{RESULTS}

There were 51 eligible neonates, all of whom were included in the study. All 51 of neonates were intubated and ventilated. One child from group 2 was transferred from Kingston and was considered lost to follow up.

Group $1(\mathrm{n}=10 ; 4$ male $(\mathrm{m})$ and 6 female (f))

Group $2(\mathrm{n}=11 ; 6 \mathrm{~m}$ and $5 \mathrm{f})$

Group $3(\mathrm{n}=30 ; 11 \mathrm{~m}$ and $19 \mathrm{f})$

There was no statistical difference between Groups 1 and 2 or Groups 2 and 3 with the exception of surfactant use, which was by design (Tables 1 and 2).

The neonates in Group 2 received an average of 1.5 doses of surfactant compared to the neonates in Group 3 receiving surfactant who received an average of 1.9 doses.

Table 1. Comparison Between Groups 1 and 2

\begin{tabular}{lccc}
\hline & Group 1 (n=10) & Group 2 (n=11) & P Value \\
\hline Mean BW (g) & $798(480-950)$ & $823(660-980)$ & NS \\
\hline Mean GA (wks) & $26.4(23-29)$ & $26.7(24-30)$ & NS \\
\hline Male & 4 & 6 & NS \\
\hline Female & 6 & 5 & NS \\
\hline Surfactant & 0 & 11 & P<0.0001 \\
\hline Pre Natal Steroids & 3 & 8 & NS \\
\hline
\end{tabular}


Table 2. Comparison Between Groups 2 and 3

\begin{tabular}{lccc}
\hline & Group 2 (n=11) & Group 3 (n=30) & P Value \\
\hline Mean BW (g) & $823(660-980)$ & $778(519-979)$ & NS \\
\hline Mean GA (wks) & $26.7(24-30)$ & $26(24-32)$ & NS \\
\hline Male & 6 & 11 & NS \\
\hline Female & 5 & 19 & NS \\
\hline Mech. Ventilated & 11 & 30 & NS \\
\hline Surfactant & 11 & 24 & NS \\
\hline \hline
\end{tabular}

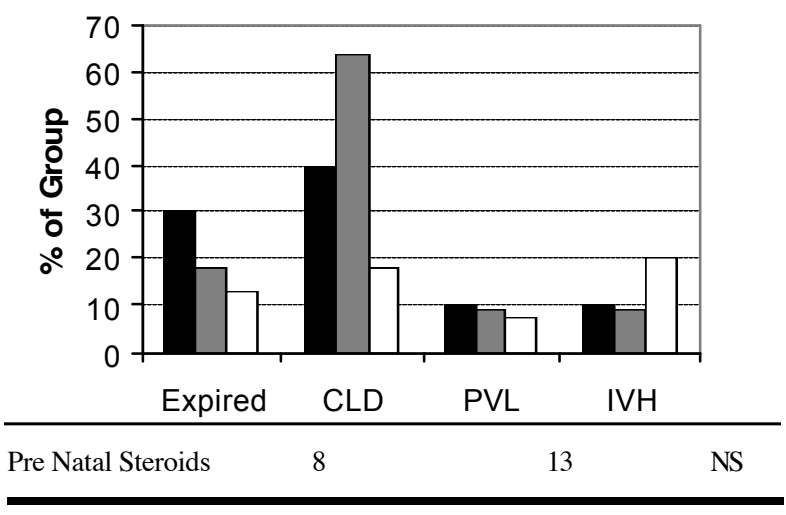

Figure 1: Outcomes in Groups 1, 2, and 3

\section{Respiratory morbidity}

In Group 1, all infants were ventilated on an intermittent mandatory mode (IMV). In this epoch,
IMV was available on both the Bear Cub and Sechrist ventilators. Median duration of mechanical ventilation was 21 days (1-109) with a standard deviation of 35.6 days. In Group 2, all infants were ventilated on IMV. Median duration of mechanical ventilation was 28 days (2-75) with a standard deviation of 20.7 days.

In Group 3, 9 infants were ventilated solely on IMV, 10 infants were ventilated solely by synchronized IMV mode (Babylog), and 11 were ventilated on a combination of IMV and/or SIMV and/or assist control (AC). The median duration of mechanical ventilation in this group was 30.0 days (1-73) with a standard deviation of 19.9 days. Table 3 records the ventilator parameters used in the three groups. Of note, there was not a significant difference in PIP and MAP between groups 1 and 2 in the first 24 hour period whereas there was a significant difference in these variables between groups 2 and 3 over the first 24 hours. There were ventilator settings recorded in Group 3 that were not noted in the earlier time periods. These include inspiratory time (IT) and inspiratory to expiratory ratio (I:E). The inspiratory times for the first, second, and third 24 hours periods were 0.47 (0.40-0.53), 0.47 (0.42-0.55), and $0.48(0.42-0.55)$ respectively. The I:E for the first, second, and third 24 hour periods were 3.5 (1.9-10.4), 4.7 (1.6-10.7), and 3.5 (1.2-10.7) respectively. Tables 4 and 5 compare duration of ventilation and oxygen dependence between the three study groups.

\section{Morbidity and mortality}

In the pre-surfactant era Group 1, 30.0\% (3 of 10)

Table 3. Ventilator Parameters in Groups 1, 2, and 3

\begin{tabular}{|c|c|c|c|c|}
\hline & Group number & 0-24 Hours & 24-48 Hours & 48-72 Hours \\
\hline PIP & $\begin{array}{l}1 \\
2 \\
3\end{array}$ & $\begin{array}{l}25.5(18-35) \\
23(18-30) \\
18(13.5-24) \#\end{array}$ & $\begin{array}{l}26.8(18-43) \\
20(14-23) \\
16(12-22)\end{array}$ & $\begin{array}{l}30.0(20-43) \\
20(18-25)^{*} \\
16(12-23) \#\end{array}$ \\
\hline MAP & $\begin{array}{l}1 \\
2 \\
3\end{array}$ & $\begin{array}{l}10.5(5.5-12.5) \\
9(7-21) \\
7.6(4-10.5) \#\end{array}$ & $\begin{array}{l}12(7.5-17) \\
7(6-10)^{*} \\
6.8(3-11)\end{array}$ & $\begin{array}{l}12(9-16) \\
6.5(6-10)^{*} \\
7.2(3-12)\end{array}$ \\
\hline $\mathbf{R R}$ & $\begin{array}{l}1 \\
2 \\
3\end{array}$ & $\begin{array}{l}55(15-95) \\
35(20-60) \\
27.5(15-40) \#\end{array}$ & $\begin{array}{l}67.5(30-130) \\
22(10-60) \\
22.5(10-45)\end{array}$ & $\begin{array}{l}72.5(36-120) \\
35(10-45) \\
30(10-60)\end{array}$ \\
\hline PEEP & $\begin{array}{l}1 \\
2 \\
3\end{array}$ & $\begin{array}{l}5(4-5) \\
5(4-6) * \\
5(4-5)\end{array}$ & $\begin{array}{l}5(4-6) \\
5(4-6)^{*} \\
5(4-7)\end{array}$ & $\begin{array}{l}4.5(4-6) \\
5(4-5)^{*} \\
5(3-6)\end{array}$ \\
\hline $\mathrm{CO}_{2}$ & $\begin{array}{l}1 \\
2 \\
3\end{array}$ & $\begin{array}{l}44.5(25-56) \\
37(26-69) \\
37.1(29.7-45.9)\end{array}$ & $\begin{array}{l}49(33-58.5) \\
38(30-50)^{*} \\
43.1(32.2-73.3)\end{array}$ & $\begin{array}{l}45(34-62) \\
39(30-48) \\
38.8(32.0-63.6)\end{array}$ \\
\hline
\end{tabular}

* significant difference between groups 1 \& 2

\# significant difference between groups $2 \& 3$ 
infants did not survive to discharge. The mean age at death in these infants was 10 (1-18) days. The cause of death was respiratory failure in all three cases. In Group 2 (ie since the introduction of surfactant use) $18.1 \%$ (2 of 11) of infants did not survive to discharge. The mean age at death in this group was 4.5 (2-7) days. Causes of death were respiratory failure and pulmonary hypoplasia. In Group 3, 16.7\% (5 of 30) of infants expired. The mean age at death in this group was 23.3 (2-64) days. Causes of death included periventricular leukomalacia, intraventricular hemorrhage, necrotizing enterocolitis, candida sepsis, gram negative sepsis, and respiratory failure. Figure 1 illustrates the comparisons of these outcomes amongst the three study groups.

Table 4. Duration of Oxygen Dependence and Ventilation (Groups 1 and 2)

\begin{tabular}{lccc}
\hline & Group 1 & Group 2 & P Value \\
\hline Median O2 Dep (d) & $45(1-148)$ & $58(2-145)$ & 0.8 \\
\hline $\begin{array}{r}\text { Median O2 Dep (CGA) } \\
34(23-47)\end{array}$ & $36(26-65)$ & 0.3 \\
\hline $\begin{array}{r}\text { Median Duration of Mech Vent (d) } \\
21 \quad(1-109)\end{array}$ & 28 & $(2-75)$ & 0.7 \\
\hline
\end{tabular}

Table 5. Duration of Oxygen Dependence and Ventilation (Groups 2 and 3)

\begin{tabular}{llll}
\hline & Group 2 & Group 3 & P Value \\
\hline Median O2 Dep (d) & $58(2-145)$ & $34.5(1-73)$ & 0.04 \\
\hline $\begin{array}{r}\text { Median O2 Dep (CGA) } \\
36(26-65)\end{array}$ & $32(26-41)$ & 0.07 \\
\hline $\begin{array}{r}\text { Median Duration of Mech Vent (d) } \\
28 \quad(2-75)\end{array}$ & $30(1-73)$ & 0.7 \\
\hline
\end{tabular}

\section{DISCUSSION}

While the introduction of surfactant has significantly reduced the mortality from respiratory distress syndrome, chronic lung disease remains a significant problem in the extremely low birth weight infant (20.7\% in 1999). By comparing extremely low birth weight infants who received surfactant (Group 2) versus those who did not (Group 1), it is possible to determine the effect of surfactant in decreasing the morbidity and mortality from respiratory distress syndrome. The observation between the two groups of patients born since the routine use of surfactant, i.e. one early in the surfactant era (Group 2) and a second cohort born ten years later (Group 3) were made in order to determine how changes in ventilatory management may affect neonatal outcome. It is clear that surfactant therapy rapidly changes lung mechanics by improving pulmonary compliance. However, it is evident that surfactant therapy alone does not decrease the incidence of chronic lung disease $(40.0 \%$ incidence in Group 1 vs $77.8 \%$ incidence in Group 2). In group 1, higher PIP and MAP was required in order to overcome the very low compliance of the surfactant deficient lung. It is likely that the high pressures used contributed significantly to the incidence of chronic lung disease in this group. In group 2, the PIP and MAP were not significantly lower in the first 24 hours. While mortality was significantly lower, the incidence of CLD is substantially higher in the infants immediately following the introduction of surfactant (Group 2), when compared to Group 1. Although surfactant therapy improved lung compliance, in the absence of a significant decrease in pressures in the first 24 hours, damage to the lung occurred. This illustrates how even brief periods of a mechanical ventilatory strategy on CMV may cause lung injury and affect outcome in the presence of changing pulmonary compliance. In comparison to Group 2, the infants in Group 3 had a significantly lower mortality rate and incidence of CLD. This likely reflects the impact of a change in ventilator management given that there were no other significant baseline differences between these two groups. Evolution in ventilation strategies included an increase in the use of SIMV, lower PIP, and lower MAP. It is of significance that the PIP and MAP were statistically lower over the first twenty-four hour time period in Group 3. The lower PIP and MAP in Group 3, combined with advances in pressure/volume limitation, has the effect of limiting barotraumas and hence reducing the incidence of CLD. The duration of $\mathrm{O} 2$ dependence is also significantly lower in Group 3. This may reflect improvements in strategies to optimize lung recruitment. The fluid management regime and the incidence of patent ductus arteriosis were not reviewed during this study and should be included in future studies.

Following the results of this study, our question remains: how can CMV and surfactant therapy continue to be optimized? Certainly, all clinicians are aware that of the need to limit volutrauma. Proven strategies include minimizing the MAP, limiting the transalveolar pressure, using small amplitudes ( $\mathrm{Vt} 4-6 \mathrm{ml} / \mathrm{kg}$ ) and the use of high frequency oscillatory ventilation. However, are there other strategies that we can utilize during CMV that can further optimize patient outcome? Recent animal and human studies show that CMV may perhaps be just as effective as HFOV when an appropriate "protective lung strategy" is used $(17,18,19)$. Froese et al. showed that early lung volume optimization preserves surfactant life span by both decreasing the rate of inactivation and allowing for endogenous production (20). From these data, it seems that a "lung 
protective strategy" with early volume optimization and surfactant therapy is an effective approach. Possible strategies include using a high respiratory rate and hence inducing an auto PEEP, determining and implementing the optimal PEEP, early permissive hypercapnea, and using shorter, more physiological inspiratory times. Recent advances in ventilator technology such as pressure support, volume guarantee, volume support, and proportional assist modes are designed to limit lung injury in the VLBW neonatal population. A further approach may be to avoid mechanical ventilation altogether and use NCPAP as is suggested by the Scandinavian studies $(7,21)$.

\section{CONCLUSION}

From these data we can conclude that while surfactant therapy has proven benefits in improving pulmonary mechanics, and hence the survival of neonates, equally important is the ventilation strategy used on the premature lung with changing compliance. From this study, it appears that surfactant therapy actually increased the risk of CLD in 1990-1991. Early ventilation strategies during the apparently stable "honeymoon period" in a patient with RDS has significant implications on long term morbidity. As demonstrated by this study, a particular crucial time is in the immediate period following surfactant administration. The learning curve experienced by clinicians in the years following the widespread use of exogenous surfactant has demonstrated a change towards acting on clinically apparent changes in the neonate, rather than a dependence on blood gas measurements and chest radiograph findings. This has been aided by more recent advances in ventilator technology (tidal volume and minute ventilation measurements) and non-invasive monitoring (transcutaneous $\mathrm{CO} 2$ measurements). The difficulty, as demonstrated by the "open-lung" studies by NIH, etc. in patients with ARDS, is to conduct randomized controlled trials comparing strategies in CMV in the neonate, in light of our evolving experience on how best to use a conventional ventilator $(13,15)$. The goal now is to overcome these challenges and to continue to learn how best to utilize a "conventional" mode of ventilation to optimize lung volume and protect the immature lung.

\section{REFERENCES}

1. McGettigan MC, Adolph VR, Ginsberg HG, Goldsmith JP. New Ways to Ventilate Newborns in Acute Respiratory Failure. Pediatric Clinics of North America 1998; 45 (3): 476-509.

2. Webb HH, Tierney DF. Experimental pulmonary edema due to intermittent positive pressure ventilation with high inflation pressures. Protection by positive end-expiratory pressure. American Review of Respiratory Disease 1974; 110 (5): 556-65.

3. Bernstein G, Mannino FL, Heldt GP, et al. Randomized multicenter trial comparing synchronized and conventional intermittent mandatory ventilation in neonates. Journal of Pediatrics 1996; 128 (4): 453-463.

4. Bernstein G. Syncrynous and patient-triggered ventilation in newborns. Neonatal Respiratory Diseases 3 1993; 6(5): 54-8, 66.

5. Van Marter LJ, Allred EN, Pagano M, et al. Do Clinical Markers of Barotrauma and Oxygen Toxicity Explain Interhospital Variation in Rates of Chronic Lung Disease? Pediatrics 2000; 105 (6): 1194-1201.

6. Avery ME; Tooley WH; Keller JB, et al. Is chronic lung disease in low birth weight infants preventable? A survey of eight centers. Pediatrics 1987; 79 (1): 26-30.

7. Jonsson B, Katz-Salamon M, Faxelius G, et al. Neonatal care of very-low-birth weight infants in special-care units and neonatal intensive-care units in Stockholm. Early nasal continuous positive airway pressure versus mechanical ventilatin: gains and losses. Aca Paediatr Suppl 1997; 419: 4-10.

8. Kuroki Y, Voelker DR. Pulmonary surfactant proteins. Journal of Biological Chemistry 1994; 269 (42): 25943-25946.

9. The HIFI Study Group. High-frequency oscillatory ventilation compared with conventional mechanical ventilation in the treatment of respiratory failure in preterm infants. New England Journal of Medicine 1989; 320 (2): 88-93.

10. Gerstmann DR, Minton SD, Stoddard RA, et al. The Provo multicenter early high-frequency oscillatory ventilation trial: improved pulmonary and clinical outcome in respiratory distress syndrome. Pediatrics 1994; 98(6 Pt 1) 1044-1057.

11. Rimensberger PC, Beghetti M, Hanquinet S, Berner M. First intention high-frequency oscillation with early lung volume optimization improves pulmonary outcome in very low birth weight infants with respiratory distress syndrome. Pediatrics 2000; 105(6): 1202-1208.

12. Courtney SE, Durand DJ, Asseliln JM et al. High Frequency Oscillatory Ventilation versus Conventional Mechanical Ventilation for Very-Low-Birth-Weight Infants. New England Journal of Medicine 2002; 347 (9): 643-652.

13. Amato MB, Barbas CS, Medeiros DM, et al. Effect of a protective-ventilation strategy on mortality in the acute respiratory distress syndrome. New England Journal of Medicine 1998; 338: 347-354.

14. National Institutes of Health. NHLBI clinical trial stopped early: successful ventilator strategy found for intensive care patients on life support. National Institutes of Health news release. March 15, 1999.

15. Stewart TE, Meade MO, Cook DJ, et al. Evaluation of a ventilation strategy to prevent barotrauma in patients at high risk for acute respiratory distress syndrome. Pressure- and VolumeLimited Ventilation Strategy Group. New England Journal of Medicine 1998; 338(6): 355-361.

16. Vazquez de Anda GF, Gommers D, Verbrugge SJ. Mechanical ventilation with high positive end-expiratory pressure and small driving pressure amplitude is as effective as high-frequency oscillatory ventilation to preserve the function of exogenous surfactant in lung-lavaged rats. Critical Care Medicine 2000; 28 (8): 2921-2925.

17. Thome U, Kössel H, Lipowsky G, et al. Randomized comparison of high-frequency ventilation with high-rate intermittent positive pressure ventilation in preterm infants with respiratory failure. Journal of Pediatrics 1999; 135 (1): 39-46.

18. Johnson AH, Peacock JL, Greenough A, et al. High Frequency Oscillatory Ventilation for the Prevention of Chronic Lung Disease of Prematurity. New England Journal of Medicine 2002; 347 (9): 633-642.

19. Stark AR. High Frequency Oscillatory Ventilation to Prevent Bronchopulmonary Dysplasia - Are we there yet? (Editorials). New England Journal of Medicine 2002; 347 (9): 682-683. 
20. Froese $\mathrm{AB}, \mathrm{McCulloch} \mathrm{PR}$, Sugiura $\mathrm{M}$ et al. Optimizing alveolar expansion prolongs the effectiveness of exogenous surfactant therapy in the adult rabbit. American Review of Respiratory Disease 1993; 148 (3): 569-577.

21. Blennow M, Jonsson B, Dahlström A, et al. [Lung function in premature infants can be improved. Surfactant therapy and CPAP reduce the need of respiratory support]. Lakartidningen 1999; 96 (13): 1571-1576.

Shaun Morris is a resident in Pediatrics at The Hospital for Sick Children and The University of Toronto. He completed his undergraduate and medical studies at Queen's University and a Master of Public Health degree at Johns Hopkins University. Research interests include infectious diseases, vaccine science \& policy, socio-economic determinants of health, and health \& human rights. He has done extensive clinical and research work in Africa, Southeast Asia, and South America and is a member of the Delta Omega Honour Society for Public Health.

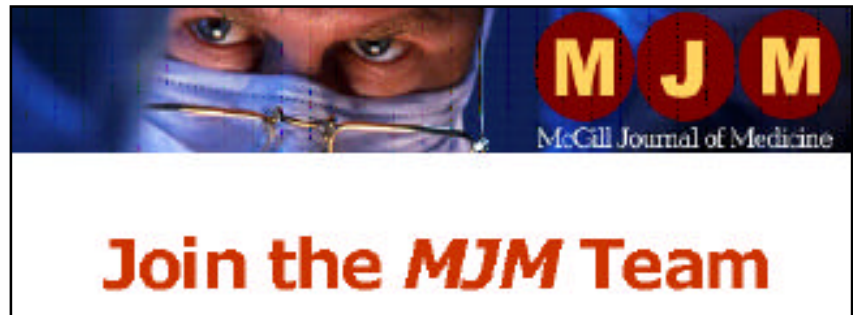

The MJM is completely student-run and is published in Montreal, Canada. As an international journal, we are always striving to add diversity to our editorial board and operations staff. MJM representatives assist students at their university to submit their work to the MJM. Contact us at mjm.med@mcgill.ca for more information about joining the MJM team.

\section{Represent the $\boldsymbol{M J M}$ at your university!}

We are seeking representatives from universities and institutes across the world to promote the journal and help us with our educational goals and international student initiatives. If you are interested in becoming a part of the MJM, please submit a brief letter of intent to mjm.med@mcgill.ca. You will join a team of over 70 staff from more than 10 countries.

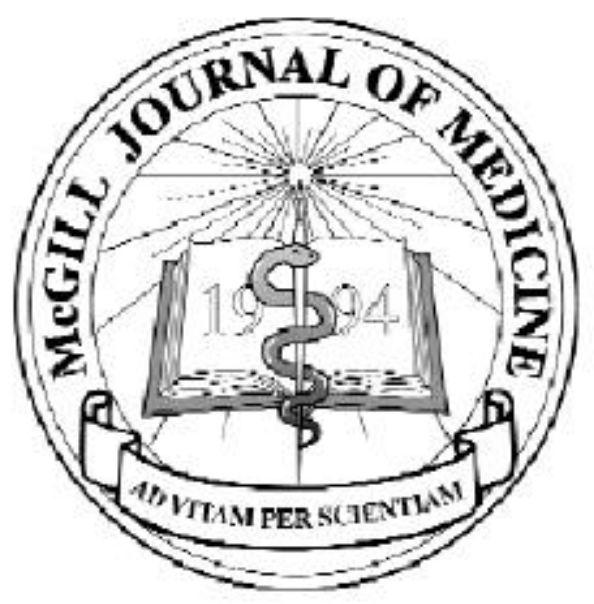

